\author{
PETTERI MANNERSALO, ${ }^{*}$ \\ VTT Technical Research Centre of Finland, Helsinki, Finland \\ ILKKA NORROS, ${ }^{* *}$ \\ VTT Technical Research Centre of Finland, Helsinki, Finland \\ RUDOLF H. RIEDI, ${ }^{* * *}$ \\ Rice University, Houston Texas, U.S.A.
}

\title{
MULTIFRACTAL PRODUCTS OF STOCHASTIC PROCESSES: CONSTRUCTION AND SOME BASIC PROPERTIES
}

\begin{abstract}
In various fields, such as teletraffic and economics, measured time series have been reported to adhere to multifractal scaling. Classical cascading measures possess multifractal scaling, but their increments form a non-stationary process. To overcome this problem we introduce a construction of random multifractal measures based on iterative multiplication of stationary stochastic processes, a special form of T-martingales. We study $\mathcal{L}^{2}$-convergence, non-degeneracy and continuity of the limit process. Establishing a power law for its moments we obtain a formula for the multifractal spectrum and hint at how to prove the full formalism.

Keywords:
\end{abstract}

Stochastic processes, random measures, multifractals, teletraffic modeling

AMS 2000 Subject Classification: Primary 60G57

Secondary 60G30

\footnotetext{
* Postal address: VTT Information Technology, P.O. Box 1202, FIN-02044 VTT, Finland

* Email address: petteri.mannersalo@vtt.fi

** Postal address: VTT Information Technology, P.O. Box 1202, FIN-02044 VTT, Finland

** Email address: illka.norros@vtt.fi

*** Postal address: ECE Dept, Rice University MS 380, Houston TX 77251-1892, USA

*** Email address: riedi@ rice.edu
} 


\section{Introduction}

This study is strongly motivated by the search of new models for teletraffic. In a sequel of papers $[28,19,22,27,9]$ it has been demonstrated that teletraffic has a very rich scaling structure when the measured traffic traces are looked at the very finest resolutions, usually resolutions $100 \mathrm{~ms}$ and smaller. More precisely, let $A(t, s)$ denote the amount of traffic (bytes) arriving on interval $[t, s)$. Empirical studies of different network environments suggest that the scaling law

$$
\log \mathrm{E} A\left(t, t+\delta_{n}\right)^{q} \approx c(q) \log \delta_{n}+C_{q}
$$

holds over a wide range of resolutions $\delta_{n}$ with a non-linear function $c(q)$. In other words, the large-deviation based multifractal spectrum seems to be non-trivial. As an illustrative example, we consider a traffic sample measured in an international link of the Finnish University and Research Network (Funet). An IP traffic trace and the corresponding empirical multiscaling moment plots are shown in Figure 1. Also in this case, multifractal-type behavior is seen. Currently, there is no complete physical understanding which and how network elements result in this phenomenon. However, the most probable candidate is the joint dynamics of TCP (Transmission Control Protocol) and queues acting in a extremely heterogeneous environment.
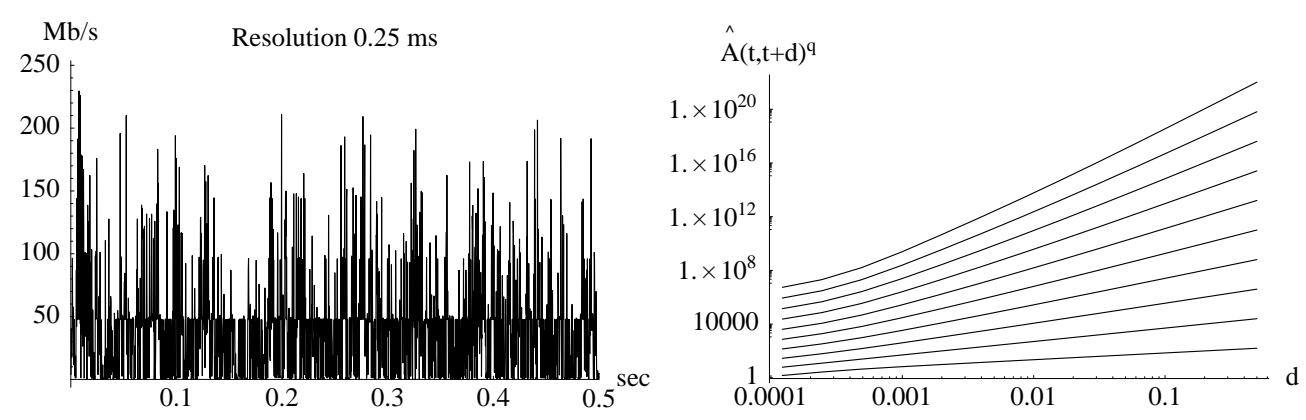

FIGURE 1: A traffic trace from an international link of FUNET and the corresponding empirical multiscaling moments with $q=0.5,1, \ldots, 5$, starting from the bottom

There are many ways to construct random multifractal measures varying from the simple binomial measures to measures generated by random branching processes (see e.g., [21, 26, $1,5,2,25,27])$. In teletraffic modeling, we would like to have, in addition to a simple and causal construction, also stationarity, i.e., stationarity of the increments of the process $A(0, t)$. Unfortunately, most of the 'classical' multifractal models, in particular tree-based 
cascades, lack both of these properties. It should be noted that a multifractal process with stationary increments is not a completely new idea. Jaffard $[12,13]$ showed that certain Lévy processes are also multifractal. However, since Lévy processes have linear multifractal spectra and real data traffic exhibits strictly concave spectra, [28, 19, 22, 27] they are unsuitable for our modeling needs. Processes with stationary increments have also been used by Gupta and Waymire [10] in the context of scaling and multiplicative structures, yet with different approach and objectives.

Research on multiplicative cascades has been very active. Especially, Mandelbrot's martingale [20,21], a simple tree-based construction with independent random multipliers, has been considered in a large number of publications; first by Kahane and Peyriére [18], and the story still continues (see e.g. [11, 5, 30, 23, 2, 3, 24]). Extensions such as relaxing the independence assumption of the multipliers or randomizing the number of offsprings have also been studied. To give a short list without intention of being complete we refer to Molchan [23] and Waymire and Williams [31, 32] regarding dependent multipliers, to Peyriére [26], Arbeiter [1], and Burd and Waymire [4] regarding random number of offsprings.

We consider a natural and stationary generalization of the multiplicative cascade construction. This scheme, as well as the genuine cascades, belong to the framework of the positive $T$-martingales and multiplicative chaos introduced by Kahane $[16,17,14]$. Let $\left(\Lambda^{(i)}\right)$ be a sequence of positive independent random functions (i.e. processes) defined on a compact metric space $T$ such that $\mathrm{E} \Lambda^{(i)}(t)=1$ for all $t \in T$ and consider finite products

$$
\Lambda_{n}(t)=\prod_{i=1}^{n} \Lambda^{(i)}(t) .
$$

Here $\Lambda_{n}$ is an indexed martingale, since it is a martingale for each $t \in T$. By [17], $\Lambda_{n}(t) \mathrm{d} \nu(t)$ converges weakly to a random measure $\Lambda(t) \mathrm{d} \nu(t)$ for any positive Radon measure $\nu$. Only partial answers are known regarding the convergence in $\mathcal{L}_{p}, 1 \leq p<\infty$. Special cases which have been studied include, for example, Gaussian chaos, i.e., lognormal multipliers $\Lambda^{(i)}$, by Kahane [16], Lévy chaos by Fan [6], and random Gibbs measures by Fan and Shieh [8]. Note that the $T$-martingale approach works also with random coverings (see e.g. [17, 14, 15, 7]).

In our setting, we restrict ourselves to the Lebesgue case and study convergence and related questions of the limiting measure of $\Lambda_{n}(t) \mathrm{d} t$, and in its simplest form our model is based on the multiplication of independent rescaled stationary stochastic processes $\Lambda^{(i)}(\cdot) \stackrel{\text { dist }}{=} \Lambda\left(b^{i} \cdot\right)$ which are piecewise constant (here $\stackrel{\text { dist }}{=}$ denotes equivalence in distributions). It is instructive 
to compare it to a Fourier decomposition where one represents or constructs a process by superposition of oscillations $\sin \left(\lambda_{i} t\right)$.

In multiplying rather than adding rescaled versions of a 'mother' process we obtain a process with novel properties which are best understood not in an additive analysis, but in a multiplicative one. Processes emerging from multiplicative construction schemes can easily be forced to have positive increments and exhibit typically a 'spiky' appearance. The so-called multifractal analysis describes the local structure of a process in terms of scaling exponents, accounting for the multiplicative structure.

It is tempting to view the multiplicative construction as an additive one - which opens the possibility to use linear theory - followed by an exponential. Such an approach, however, obscures what happens in the limit. As with the cascades, an infinite product of random processes will typically (almost surely at almost all times) be zero; equally, its logarithm tends to negative infinity. A non-degenerate limiting behavior can be observed for the product, though, by taking a distributional limit rather than pointwise limit. In simpler words, a multiplier $\Lambda^{(i)}(t)$ should not be evaluated in points but should be seen as redistributing or re-partitioning the mass. In the words of teletraffic modeling, $\Lambda^{(i)}(t)$ can be thought of as a local change in the arrival rate where one is interested actually in the integrated 'total load' process. Consequently, we will study

$$
A_{n}(t)=\int_{0}^{t} \prod_{i=0}^{n} \Lambda^{(i)}(s) \mathrm{d} s
$$

which converges to a well defined, non-degenerate and continuous process under suitable conditions.

The paper is structured as follows: We start by studying the construction of multifractal measures based on iterative multiplication of stochastic processes as in $A_{n}$ above, in particular convergence and non-degeneracy. Then, we consider a special case where the multipliers are independent rescaled versions of some mother process, looking at continuity, power laws of moments as well as long-range dependence (LRD) of the limiting process. Finally, we provide an application-friendly family based on piecewise constant processes with exponentially distributed sojourn times. 


\section{Multifractal products of stochastic processes}

In order to keep the presentation simple, we only consider 1-dimensional processes on the closed unit interval $T=[0,1]$. Extensions to the real line $\mathbb{R}$ as well as to higher dimensions are not too difficult.

\subsection{Construction}

We start from $T$-martingales defined by independent multiplication as in [17]. Consider a family of independent positive processes $\left\{\Lambda^{(i)}(t)\right\}_{t \in T}$ with

$$
\mathrm{E}^{(i)}(t)=1 \quad \forall t \in T, i=0,1,2 \ldots
$$

Later, when studying particular properties of the process, we will usually assume that the $\Lambda^{(i)}$ 's are stationary, but this is not necessary in the general case.

Define the finite product processes

$$
\Lambda_{n}(t) \doteq \prod_{i=0}^{n} \Lambda^{(i)}(t)
$$

and the corresponding cumulative processes

$$
A_{n}(t) \doteq \int_{0}^{t} \Lambda_{n}(s) \mathrm{d} s=\int_{0}^{t} \prod_{i=0}^{n} \Lambda^{(i)}(s) \mathrm{d} s, \quad n=0,1, \ldots
$$

Sometimes, it is easier to consider the corresponding positive measures defined on the Borel sets $\mathcal{B}$ of $T$ :

$$
\mu_{n}(B) \doteq \int_{B} \Lambda_{n}(s) \mathrm{d} s, \quad n=0,1, \ldots, \quad B \in \mathcal{B} .
$$

\subsection{Convergence}

Theorem 1. [17] The random measures $\mu_{n}$ converge weakly a.s. to a random measure $\mu$. Moreover, given a finite or countable family of Borel sets $B_{j}$ on $T$, we have with probability one:

$$
\forall j \quad \mu\left(B_{j}\right)=\lim _{n \rightarrow \infty} \mu_{n}\left(B_{j}\right) .
$$

The a.s. convergence of $A_{n}(t)$ in countably many points at the same time can be extended to all points in $T$ if we know that the limit process $A$ is almost surely continuous. Conditions for continuity in a more specific setup are given later in Proposition 4. 
Corollary 1. If $A_{n} \rightarrow A$ weakly a.s., and $A_{n}$ and $A$ are continuous almost surely, then with probability one: $\forall t \in T, A(t)=\lim _{n \rightarrow \infty} A_{n}(t)$.

Unfortunately, Theorem 1 does not say anything about the $\mathcal{L}_{1}$-convergence; as is noted in [17], one of the following two cases is met: either $A_{n}(t) \rightarrow A(t)$ in $\mathcal{L}_{1}$ for each given $t$ or $A_{n}(1)$ converges to 0 almost surely. The cases are called non-degenerate and degenerate, respectively. In this paper, we present conditions for $\mathcal{L}_{2}$-convergence which is the easiest to handle.

Proposition 1. Suppose that the stationary independent processes $\Lambda^{(i)}, i=0,1, \ldots$, satisfy

$$
\begin{aligned}
\operatorname{E} \Lambda^{(i)}(t) & =1, \quad \forall t \in T, \\
\operatorname{Var} \Lambda^{(i)}(t) & =\sigma^{2}<\infty, \quad \forall t \in T, \\
\operatorname{Cov}\left(\Lambda^{(i)}\left(t_{1}\right), \Lambda^{(i)}\left(t_{2}\right)\right) & =\sigma^{2} \rho_{i}\left(t_{1}-t_{2}\right), \forall t_{1}, t_{2} \in T,
\end{aligned}
$$

where $\rho_{i}, i=0,1, \ldots$, are the normalized covariance functions with $\rho_{i}(0)=1$. Then $\left(A_{n}(1)\right.$ : $n \in \mathbb{N}$ ) is bounded in $\mathcal{L}_{2}$ if and only if

$$
\sum_{n=0}^{\infty} a_{n}(1)<\infty
$$

where $a_{n}(t)=\int_{0}^{t}(t-s) \rho_{n}(s) \prod_{i=0}^{n-1}\left(1+\sigma^{2} \rho_{i}(s)\right) \mathrm{d} s$. Furthermore, if condition (4) holds true then $A_{n}(t) \rightarrow A(t)$ in $\mathcal{L}_{2}$ for all $t \in[0,1]$.

Note: Similarly as in Theorem 1, we could prove the simultaneous convergence for points in a countable set. In order to have $\mathcal{L}_{2}$ convergence for all points in $T$ at the same time, however, we need extra conditions, like the continuity of $A$ which we will address in the following section.

Proof. By Fubini, $\left\{A_{n}(1)\right\}$ is a martingale with respect to $\left\{\left(\mathcal{F}_{n}, P\right)\right\}$, where

$$
\mathcal{F}_{n}=\sigma\left(\Lambda^{(0)}, \Lambda^{(1)}, \ldots, \Lambda^{(n)}\right)
$$

Since $A_{n}(1) \in \mathcal{L}_{2}$ for any fixed $n$, it is enough to check if $\sum \mathrm{E}\left[\left(A_{k}(1)-A_{k-1}(1)\right)^{2}\right]<\infty$. 
By the definition of $A_{n}$ and assumptions (1)-(3)

$$
\begin{aligned}
\mathrm{E} & {\left[\left(A_{n}(1)-A_{n-1}(1)\right)^{2}\right] } \\
& =\mathrm{E} \int_{0}^{1} \int_{0}^{1}\left(\Lambda^{(n)}\left(s_{1}\right)-1\right)\left(\Lambda^{(n)}\left(s_{2}\right)-1\right) \Lambda_{n-1}\left(s_{1}\right) \Lambda_{n-1}\left(s_{2}\right) \mathrm{d} s_{1} \mathrm{~d} s_{2} \\
& =\int_{0}^{1} \int_{0}^{1} \operatorname{Cov}\left(\Lambda^{(n)}\left(s_{1}\right), \Lambda^{(n)}\left(s_{2}\right) \mathrm{E}\left(\Lambda_{n-1}\left(s_{1}\right) \Lambda_{n-1}\left(s_{2}\right)\right) \mathrm{d} s_{1} \mathrm{~d} s_{2}\right. \\
& =\int_{0}^{1} \int_{0}^{1} \sigma^{2} \rho_{n}\left(\left|s_{1}-s_{2}\right|\right) \prod_{i=0}^{n-1}\left(1+\sigma^{2} \rho_{i}\left(\left|s_{1}-s_{2}\right|\right) \mathrm{d} s_{1} \mathrm{~d} s_{2}\right. \\
& =2 \sigma^{2} \int_{0}^{1}(1-s) \rho_{n}(s) \prod_{i=0}^{n-1}\left(1+\sigma^{2} \rho_{i}(s)\right) \mathrm{d} s .
\end{aligned}
$$

Thus $\left(A_{n}(1): n \geq 0\right)$ is bounded in $\mathcal{L}_{2}$ if and only if

$$
\sum_{n=0}^{\infty} \int_{0}^{1}(1-x) \rho_{n}(x) \prod_{i=0}^{n-1}\left(1+\sigma^{2} \rho_{i}(x)\right) \mathrm{d} x<\infty .
$$

Since $A$ is a positive, nondecreasing process, the above condition is sufficient for $A_{n}(t)$ being bounded in $\mathcal{L}_{2}$ for all $t \in[0,1]$.

Explicit knowledge of the decay rates of the covariance functions simplifies the $\mathcal{L}_{2}$-condition considerably. The following result covers a fair range of correlation functions:

Corollary 2. If there are positive constants $\nu, \gamma, b$ and $C$ such that

$$
\exp \left(-\nu\left|b^{i} s\right|\right) \leq \rho_{i}(s) \leq\left|C b^{i} s\right|^{-\gamma}
$$

for all $s \in[0,1]$, then $\left(A_{n}(t): n \geq 0\right)$ is bounded in $\mathcal{L}_{2}$ if and only if

$$
b>1+\sigma^{2} .
$$

The particular form of (5) is motivated by the processes we study in Section 3. Notice that the condition $b>1+\sigma^{2}$ is the same as for Mandelbrot's martingale, as is shown in [18].

Proof. Sufficiency: Because $\rho_{i}(s)>0$ (i.e., $\Lambda^{(i)}$ are positively correlated),

$$
a_{n}(t) \leq \int_{0}^{t} \rho_{n}(s) \prod_{i=0}^{n-1}\left(1+\sigma^{2} \rho_{i}(s)\right) \mathrm{d} s .
$$


Without losing generality, we can set $t=1$ and assume that $C=1, \gamma \in(0,1)$ and $1+\sigma^{2}<$ $b<\left(1+\sigma^{2}\right)^{1 / 1-\gamma}$. First, we split the integration into parts and use the fact that $\rho_{i}(s) \leq 1$ for all $s$. Let $\overline{\rho_{i}}(s)=\min \left\{1,\left|b^{i} s\right|^{-\gamma}\right\}$ and take an arbitrary $m<n$, then

$$
\begin{aligned}
& a_{n}(1)=\int_{0}^{1} \rho_{n}(s) \prod_{i=0}^{n-1}\left(1+\sigma^{2} \rho_{i}(s)\right) \mathrm{d} s \\
& \quad \leq \int_{0}^{b^{-n+m}}\left(1+\sigma^{2}\right)^{n} \mathrm{~d} s+\sum_{i=m}^{n-1}\left(1+\sigma^{2}\right)^{n+m-i} \int_{b^{-n+i}}^{b^{-n+i+1}} \bar{\rho}_{n}(s) \prod_{j=n+m-i}^{n-1}\left(1+\sigma^{2} \bar{\rho}_{j}(s)\right) \mathrm{d} s \\
& \quad \leq\left(1+\sigma^{2}\right)^{n}\left\{b^{-n+m}+\sum_{i=0}^{n-m-1} \int_{b^{-n+m+i}}^{b^{-n+m+i+1}} \bar{\rho}_{n}(s)\left[\frac{1+\sigma^{2} \bar{\rho}_{n-i}\left(b^{-n+m+i}\right)}{1+\sigma^{2}}\right]^{i} \mathrm{~d} s\right\} \\
& =\left(1+\sigma^{2}\right)^{n}\left\{b^{-n+m}+\sum_{i=0}^{n-m-1} \int_{b^{-n+m+i}}^{b^{-n+m+i+1}} b^{-n \gamma} s^{-\gamma}\left[\frac{1+\sigma^{2} b^{-m \gamma}}{1+\sigma^{2}}\right]^{i} \mathrm{~d} s\right\} \\
& \quad=\left(1+\sigma^{2}\right)^{n} b^{-n}\left\{b^{m}+\frac{\left(b^{1-\gamma}-1\right) b^{(1-\gamma) m}}{1-\gamma} \sum_{i=0}^{n-m-1}\left[b^{1-\gamma} \frac{1+\sigma^{2} b^{-m \gamma}}{1+\sigma^{2}}\right]^{i}\right\}
\end{aligned}
$$

where the second inequality holds because $\bar{\rho}_{i}(s)$ is decreasing with respect to both $s$ and $i$. Next, let $m=\min \left\{k \in \mathbb{N}: b^{1-\gamma} \frac{1+b^{-k \gamma} \sigma^{2}}{1+\sigma^{2}}<1\right\}$. Then

$$
a_{n}(1) \leq C(b, m) b^{-n}\left(1+\sigma^{2}\right)^{n}
$$

where $C(b, m)<\infty$ does not depend on $n$. Thus $\sum a_{n}(1)<\infty$.

Necessity: Let $\delta, t>\delta>0$, be arbitrary. Then

$$
\begin{aligned}
a_{n}(t) & \geq \delta \int_{0}^{t-\delta} e^{-b^{n} \nu s}\left(1+\sigma^{2} e^{-\nu b^{n} s}\right)^{n} \mathrm{~d} s \\
& =\frac{\left(1+\sigma^{2}\right) \delta}{\nu}\left(1-\frac{\left(1+\sigma^{2} e^{-\nu b^{n}(t-\delta)}\right)^{n+1}}{\left(1+\sigma^{2}\right)^{n+1}}\right) \frac{\left(1+\sigma^{2}\right)^{n}}{(n+1) b^{n}} \\
& \geq \frac{\left(1+\sigma^{2}\right) \delta}{2 \nu} \frac{\left(1+\sigma^{2}\right)^{n}}{(n+1) b^{n}},
\end{aligned}
$$

if $n$ is large enough. Thus $\sum \tilde{a}_{n}(t)$ diverges if $b \leq 1+\sigma^{2}$.

\section{Self-similar Products}

\subsection{Invariance and Convergence}

The analysis of the limiting process $A(t)$ simplifies greatly provided that the multipliers $\Lambda^{(i)}$ are connected through a rescaling property. More precisely, throughout this section we 
assume that the processes $\Lambda^{(i)}$ are independent rescaled copies of some stationary mother process $\Lambda$, i.e.,

$$
\Lambda^{(i)}(\cdot) \stackrel{\text { dist }}{=} \Lambda\left(b^{i} \cdot\right)
$$

where $b>1$ and $\mathrm{E} \Lambda=1$. Two results follow straight from this rescaling property:

Corollary 3. Assume self-similar multipliers in the sense of (7) with a mother process $\Lambda$ which lies in $\mathcal{L}_{2}$ and such that its scaled covariance function satisfies

$$
\exp (-\nu|x|) \leq \rho(x) \leq|C x|^{-\gamma}
$$

for some positive numbers $\nu$ and $\gamma$. Then, $A_{n}(t)$ converges in $\mathcal{L}_{2}$ if and only if $b>1+\sigma^{2}$, where $\sigma^{2}=\operatorname{Var} \Lambda$.

Proposition 2. Assume (7) and that $A_{n}(t)$ converges in $\mathcal{L}_{1}$. Then, the limit process A satisfies the recursion

$$
A(t)=\frac{1}{b} \int_{0}^{t} \Lambda(s) \mathrm{d} \tilde{A}(b s)
$$

where

(i) the processes $\Lambda$ and $\tilde{A}$ are independent, and

(ii) the processes $A$ and $\tilde{A}$ are equally distributed.

To relate to the classical cascades, let us note that 'Mandelbrot's martingale' [18] can formally be written exactly as (7) with the only difference that $\Lambda$ has to be chosen nonstationary - it is constant over the intervals $[k / b,(k+1) / b)(k$ integer $)$ - and the values $\Lambda(k / b)$ form a sequence of i.i.d. random variables of mean 1 . In this case, (8) may be reduced to

$$
\mathcal{A}(1 / b)=\frac{1}{b} \Lambda(0) \tilde{\mathcal{A}}(1),
$$

where the random variables $\Lambda(0)$ and $\tilde{\mathcal{A}}(1)$ are independent, and $\tilde{\mathcal{A}}(1)$ is equally distributed as $\mathcal{A}(1)$.

Generalizing (9) to $\mathcal{A}(k / b)-\mathcal{A}((k-1) / b)=(1 / b) \Lambda((k-1) / b)(\tilde{\mathcal{A}}(k)-\tilde{\mathcal{A}}(k-1))$ and summing over $k=1, \ldots, b$ yields the invariance ${ }^{1}$ or recursion [18, equation 3], from which Kahane and Peyriere derive all their results. Their theorem 1 establishes four equivalent

\footnotetext{
${ }^{1}$ For cascades, the random variables $\tilde{\mathcal{A}}(k)-\tilde{\mathcal{A}}(k-1)$ are independent.
} 
conditions for non-degeneracy of the limiting measure. The counterpart to the equivalence of conditions $(\alpha),(\beta)$ and $(\gamma)$ of their theorem 1 is easy to establish using similar arguments as theirs (see Proposition 3 below). The main result of their theorem 1, however, the nondegeneracy condition $(\delta)$ in terms of the moments of $\Lambda$, remains an open problem in our case. At the moment, we can only state a sufficient and necessary condition for $\mathcal{L}_{2}$ convergence, given in Corollary 3, which is sufficient for non-degeneracy.

Proposition 3. Assuming (7), the following are equivalent:

$(\alpha)$ the a.s. martingale limit $A(1)$ satisfies $\mathrm{E} A(1)=1$;

( $\beta)$ the a.s. martingale limit $A(1)$ satisfies $\mathrm{E} A(1)>0$;

$(\gamma)$ the equation (8) has a solution $A$ such that $\mathrm{E} A(1)=1$.

Proof. Assume $(\gamma)$. Then $\tilde{A}$ can again be written as

$$
\tilde{A}(t)=\frac{1}{b} \int_{0}^{t} \tilde{\Lambda}(s) \mathrm{d} \tilde{\tilde{A}}(b s),
$$

where $\tilde{\Lambda}$ is independent from $\Lambda$, etc. Denote $\Lambda^{(0)}=\Lambda, \Lambda^{(1)}=\tilde{\Lambda}$ and so on. Then

$$
\mathrm{E}\left(A(1) \mid \Lambda^{(0)}, \ldots, \Lambda^{(n)}\right)=\int_{0}^{1} \prod_{i=0}^{n} \Lambda^{(i)}\left(b^{i} s\right) \mathrm{d} s .
$$

This martingale is uniformly integrable — but it is the same (in distribution at least) as $A_{n}(1)$ ! Thus, $\alpha$ holds. The remaining implications $\alpha \Rightarrow \beta \Rightarrow \gamma$ are obvious.

\subsection{Continuity}

The following proposition gives sufficient conditions for the continuity of the limit process. Note that, in the random cascade case [18], the non-degeneracy is equivalent with the condition $\mathrm{E} \Lambda \log \Lambda<\log b$.

Proposition 4. If, in addition to (7), there exists a non-trivial integrable limit $A=\lim _{n \rightarrow \infty} A_{n}$, $\mathrm{E} \Lambda \log \Lambda<\log b$, and $\mathrm{E} A(1) \log A(1)<\infty$, then $A$ is continuous.

Proof. Denote by $B$ the pure jump part of $A$ :

$$
B(t)=\sum_{s \leq t} \Delta A(s)
$$


By Proposition 3, (8) holds. Since no "new" jumps can be created in the integration, (8) holds for $B$ as well. Denote $g(x)=x \log x$. Now,

$$
\begin{aligned}
\mathrm{E} & \sum_{s \leq 1} g(\Delta B(s))=\mathrm{E} \sum_{s \leq 1} g\left(\frac{1}{b} \Lambda(s) \Delta \tilde{B}(b s)\right) \\
& =b \mathrm{E} \sum_{s \leq 1} g\left(\frac{1}{b} \Lambda(s / b) \Delta \tilde{B}(s)\right) \\
& =b \mathrm{E} \sum_{s \leq 1}\left[\frac{1}{b} \Lambda(s / b) \Delta B(s)\left(\log \Lambda(s / b)+\log \frac{\Delta B(s)}{b}\right)\right] \\
& =\mathrm{E} \sum_{s \leq 1} \Lambda(s / b) \Delta B(s) \log \frac{\Delta B(s)}{b}+\mathrm{E} \sum_{s \leq 1}(\Lambda(s / b) \log \Lambda(s / b)) \Delta B(s) \\
& =\mathrm{E} \sum_{s \leq 1} g(\Delta B(s))-\log (b) \mathrm{E} B(1)+\mathrm{E}(\Lambda \log \Lambda) \mathrm{E} B(1),
\end{aligned}
$$

where the second equality uses the fact that $B$ has stationary increments. Since $g$ is superadditive,

$$
\mathrm{E} \sum_{s \leq 1} g(\Delta B(s)) \leq \mathrm{E} g(B(1)) \leq \mathrm{E} g(A(1))<\infty .
$$

It follows that $\log (b) \mathrm{E} B(1)=\mathrm{E}(\Lambda \log \Lambda) \mathrm{E} B(1)$. Using $\mathrm{E} B(1) \leq \mathrm{E} A(1)<\infty$ and $\mathrm{E} \Lambda \log \Lambda<$ $\log b$, we find $\mathrm{E} B(1)=0$ and $B \equiv 0$ a.s.

\subsection{Scaling of moments}

Next we consider general moments. Assuming that the possible jumps of $\Lambda$ behave nicely enough, we can show that $\mathrm{E} A(t)^{q} \sim t^{q-\log _{b} \Lambda^{q}}$. As straightforward corollaries, this gives almost the same necessary conditions for non-degeneracy and boundedness in $\mathcal{L}_{p}$ as [18].

Proposition 5. Assume (8) and that $A$ is non-degenerate. Let $q>0$ be such that $A(1) \in \mathcal{L}_{q}$, and assume that

$$
\sum_{n=0}^{\infty} c\left(q, b^{-n}\right)<\infty
$$

where $c(q, t) \doteq \operatorname{Esup}_{s \in[0, t]}\left|\Lambda(0)^{q}-\Lambda(s)^{q}\right|$. Then there exist constants $\bar{C}$ and $\underline{C}$ such that

$$
\underline{C} t^{q-\log _{b} \mathrm{E} \Lambda^{q}} \leq \mathrm{E} A(t)^{q} \leq \bar{C} t^{q-\log _{b} \mathrm{E} \Lambda^{q}} \quad \forall t \in[0,1] .
$$

To motivate (11) we recall the simple form (9) of the invariance of $A(t)$ in the case of a cascade which implies that for cascades

$$
\mathrm{E} \mathcal{A}\left(1 / b^{n}\right)^{q}=\left(b^{-q} \mathrm{E} \Lambda^{q}\right)^{n} \mathrm{E} \mathcal{A}(1)^{q}
$$


This simple law is a direct consequence of the fact that the multipliers $\Lambda^{(i)}$ of a cascade $\mathcal{A}$ are constant over intervals of length $1 / b^{i}$. Proposition 5 claims that if the $q$-th power of the multipliers $\Lambda^{(i)}$ do not oscillate too much over these intervals (see (10)) then roughly the same scaling law (11) holds.

Proof. Let us first establish (11) for the discrete points $t_{n}=b^{-n}, n=0,1,2, \ldots$. To this end, set

$$
R_{n}(q)=\mathrm{E}\left[A\left(b^{-n}\right)^{q}\right]-\frac{\mathrm{E} \Lambda(0)^{q}}{b^{q}} \mathrm{E}\left[A\left(b^{-n+1}\right)^{q}\right] .
$$

For cascades, $R_{n}$ is zero. Here, we show that it is not too large provided (10) holds. Using equation (8) and equal distribution of $A$ and $\tilde{A}$, we find

$$
R_{n}(q)=b^{-q} \mathrm{E}\left[\left(\int_{0}^{b^{-n}} \Lambda(s) \mathrm{d} \tilde{A}(b s)\right)^{q}-\Lambda(0)^{q} \tilde{A}\left(b^{-n+1}\right)^{q}\right] .
$$

Next, we use that for positive $x$ we have

$$
\left|\left(\int_{I} x(s) \mathrm{d} \mu(s)\right)^{q}-C\right| \leq \sup _{s \in I}\left|x(s)^{q} \mu(I)^{q}-C\right| .
$$

Since $\tilde{A}\left(b^{-n+1}\right)=\int_{0}^{b^{-n}} \mathrm{~d} \tilde{A}(b s)$, we find

$$
\begin{aligned}
\left|R_{n}(q)\right| & \leq b^{-q} \mathrm{E}\left(\sup _{s \in\left[0, b^{-n}\right]}\left|\Lambda(s)^{q} \tilde{A}\left(b^{-n+1}\right)^{q}-\Lambda(0)^{q} \tilde{A}\left(b^{-n+1}\right)^{q}\right|\right) \\
& =b^{-q} \mathrm{E} \tilde{A}\left(b^{-n+1}\right)^{q} \mathrm{E} \sup _{s \in\left[0, b^{-n}\right]}\left|\Lambda(s)^{q}-\Lambda(0)^{q}\right| \\
& =b^{-q} \mathrm{E}\left[A\left(b^{-n+1}\right)^{q}\right] c\left(q, b^{-n}\right) .
\end{aligned}
$$

Let $n^{*}=\min \left\{n: c\left(q, b^{-n}\right)<\mathrm{E} \Lambda^{q}\right\}$ and assume that $n>n^{*}$. Set $v_{n}=\mathrm{E} A\left(b^{-n}\right)^{q}$ for short. Applying the definition of $R_{n}$ recursively we find

$$
\begin{aligned}
v_{n}(q) & =v_{n^{*}}(q) \prod_{i=n^{*}}^{n-1}\left(\frac{\mathrm{E} \Lambda^{q}}{b^{q}}+\frac{R_{i+1}(q)}{v_{i}(q)}\right) \\
& =v_{n^{*}}(q)\left(\frac{\mathrm{E} \Lambda^{q}}{b^{q}}\right)^{n-n^{*}} \prod_{i=n^{*}}^{n-1}\left(1+\frac{b^{q} R_{i+1}(q)}{v_{i}(q) \mathrm{E} \Lambda^{q}} .\right)
\end{aligned}
$$

The product term is the 'correction' needed because the $\Lambda^{(i)}$ are not constant over $b$-ary intervals. The product term is finite since

$$
\sum_{i=n^{*}}^{\infty} \log \left(1-\frac{c\left(q, b^{-i}\right)}{\mathrm{E} \Lambda^{q}}\right) \leq \sum_{i=n^{*}}^{n-1} \log \left(1+\frac{b^{q} R_{i+1}(q)}{v_{i}(q) \mathrm{E} \Lambda^{q}}\right) \leq \sum_{i=n^{*}}^{\infty} \log \left(1+\frac{c\left(q, b^{-i}\right)}{\mathrm{E} \Lambda^{q}}\right) .
$$


and (10) implies

$$
-\infty<\sum_{n=n^{*}}^{\infty} \log \left(1-\frac{c\left(q, b^{-n}\right)}{\mathrm{E} \Lambda^{q}}\right) \leq \sum_{n=0}^{\infty} \log \left(1+\frac{c\left(q, b^{-n}\right)}{\mathrm{E} \Lambda^{q}}\right)<\infty .
$$

Noticing that $b^{-n q}\left(\mathrm{E} \Lambda^{q}\right)^{n}=\left(b^{-n}\right)^{q-\log _{b} \mathrm{E} \Lambda^{q}}=t_{n}^{q-\log _{b} \mathrm{E} \Lambda^{q}}$ shows that there exist positive constants $C_{1}$ and $C_{2}$ such that for all $n$

$$
C_{1} t_{n}^{q-\log _{b} \mathrm{E} \Lambda^{q}} \leq \mathrm{E} A\left(t_{n}\right)^{q} \leq C_{2} t_{n}^{q-\log _{b} \mathrm{E} \Lambda^{q}}
$$

Finally, the bounds have to be extended for all $t \in[0,1]$. Since $A^{q}(\cdot)$ is a non-decreasing process, it is an easy exercise to show that a correction factor large enough is $b^{q-\log _{b} \mathrm{E} \Lambda^{q}}$. Thus

$$
\begin{aligned}
& \underline{C}=C_{1} b^{-q+\log _{b} \mathrm{E} \Lambda^{q}} \\
& \bar{C}=C_{2} b^{q-\log _{b} \mathrm{E} \Lambda^{q}}
\end{aligned}
$$

are suitable constants for inequality (11).

Corollary 4. If $A$ is non-degenerate and $\Lambda$ as in Proposition 5, then $\mathrm{E} \Lambda \log _{b} \Lambda \leq 1$.

Proof. Denote $N=\left\lceil b^{n}\right\rceil$ and $\varphi(q)=1-q+\log _{b} \mathrm{E} \Lambda^{q}$. By sub-additivity of the function $x^{q}, q \in[0,1], A(1)^{q} \leq \sum_{i=1}^{N} \mu\left(I_{i}^{(n)}\right)^{q}$, where $I_{i}^{(n)}=\left[i b^{-n},(i+1) b^{-n}\right), i=0, \ldots, N$. Applying stationarity of the increments and Proposition 5 gives

$$
\mathrm{E} A(1)^{q} \leq \bar{C} N b^{n\left(-q+\log _{b} \mathrm{E} \Lambda^{q}\right)} \leq \tilde{C} b^{n\left(1-q+\log _{b} \mathrm{E} \lambda^{q}\right)}=\tilde{C} b^{n \varphi(q)}
$$

for all $n$. This means that $\varphi(q) \geq 0$ for all $q \in[0,1]$. Since $\varphi(1)=0$, it follows that $\varphi^{\prime}\left(1^{-}\right) \leq 0$, i.e., $\mathrm{E} \Lambda \log _{b} \Lambda \leq 1$.

Corollary 5. If $A$ is non-degenerate, $A(1) \in \mathcal{L}_{q}, q>1$, and $\Lambda$ as in Proposition 5, then $\mathrm{E} \Lambda^{q} \leq b^{q-1}$.

Proof. Denote $N=\left\lfloor b^{n}\right\rfloor$. By super-additivity of the function $x^{q}, q>1, A(1)^{q} \geq$ $\sum_{i=1}^{N} \mu\left(I_{i}^{(n)}\right)^{q}$, where $I_{i}^{(n)}=\left[i b^{-n},(i+1) b^{-n}\right), i=0, \ldots, N$. Applying stationarity of the increments and Proposition 5 gives

$$
\mathrm{E} A(1)^{q} \geq \underline{C} N b^{n\left(-q+\log _{b} \mathrm{E} \Lambda^{q}\right)} \geq \tilde{C} b^{n\left(1-q+\log _{b} \mathrm{E} \Lambda^{q}\right)}
$$

for all $n$. Thus $1-q+\log _{b} \mathrm{E} \Lambda^{q} \leq 0$. 
We conjecture that $\mathrm{E} \Lambda^{q}<b^{q-1}$, together with some extra condition on the dependence structure of $\Lambda$, is sufficient to guarantee that $A(1)$ converges in $\mathcal{L}_{q}$ This is indeed true for $q=2$. If the conjecture holds, then we may conclude that $\mathrm{E} \Lambda \log _{b} \Lambda<1$, together with the existence of a finite $q^{\prime}$ th moment for some $q>1$, is sufficient to guarantee that $A$ be nondegenerate. Indeed, setting $\varphi(q)=1-q+\log _{b} \mathrm{E}^{q}$ as before, $\varphi^{\prime}(1+)<0$ implies $\varphi(q)<0$ for some $q>1$, thus the convergence of $A_{n}$ in $\mathcal{L}_{q}$ and $\mathrm{E} A(t)=\lim _{n} \mathrm{E} A_{n}(t)=t$.

In applications related to multifractals, we are usually interested in scaling properties. The deterministic partition function is defined as

$$
T(q) \doteq \liminf _{n \rightarrow \infty} \frac{\log \mathrm{E} \sum_{k=0}^{N-1} \mu\left(I_{k}^{(n)}\right)^{q}}{\log \left|I^{(n)}\right|}=\liminf _{n \rightarrow \infty}-\frac{1}{n} \log _{2} \mathrm{E} \sum_{k=0}^{N-1} \mu\left(I_{k}^{(n)}\right)^{q},
$$

where $I_{k}^{(n)}=\left[k 2^{-n},(k+1) 2^{-n}\right), k=0, \ldots, 2^{n}-1$. This may be the easiest scaling function to compute. A study of the pathwise scaling properties as well as the multifractal spectra is under way.

Corollary 6. If $A$ is non-degenerate, $A(1) \in \mathcal{L}_{q}, q>1$, and $\Lambda$ as in Proposition 5, then $T(q)=q-1-\log _{b} \mathrm{E}^{q}$.

Proof. By Proposition 5,

$$
\lim _{n \rightarrow \infty} \frac{1}{n} \log _{2} \mathrm{E} A\left(2^{-n}\right)=-q+\log _{b} \mathrm{E} \Lambda^{q} .
$$

Since $A$ has stationary increments,

$$
\begin{aligned}
T(q) & =\liminf _{n \rightarrow \infty}-\frac{1}{n} \log _{2} \mathrm{E} \sum_{k=0}^{2^{n}-1} \mu\left(I_{k}^{(n)}\right)^{q} \\
& =-\lim \frac{1}{n} \log _{2} 2^{n} \mathrm{E} A\left(2^{-n}\right)^{q} \\
& =q-1-\log _{b} \mathrm{E} \Lambda^{q} .
\end{aligned}
$$

If the original mother process is positively correlated we can show that the multiplicative construction increases variance. This implies that our construction preserves Long Range Dependence (LRD, see e.g. [29]). We say that a square integrable process $B$ with stationary increments is long range dependent if, for some $\gamma>1$, $\operatorname{Var} B(t) \geq c t^{\gamma}$ for all $t$.

Proposition 6. Assume, in addition to (7), that $A$ is non-degenerate, $A(1) \in \mathcal{L}_{2}$ and $\Lambda$ is positively correlated. Then $\operatorname{Var} A_{t} \geq \operatorname{Var} \int_{0}^{t} \Lambda(s) \mathrm{d} s$. 
Proof. Since $\Lambda$ and $\tilde{A}$ are independent, a simple manipulation and rearrangement gives

$$
\begin{aligned}
\operatorname{Var} A(t)= & \mathrm{E}\left(\frac{1}{b^{2}} \int_{0}^{t} \int_{0}^{t} \Lambda\left(s_{1}\right) \Lambda\left(s_{2}\right) \mathrm{d} \tilde{A}\left(b s_{1}\right) \mathrm{d} \tilde{A}\left(b s_{2}\right)\right)-t^{2} \\
= & \mathrm{E}\left(\frac{1}{b^{2}} \int_{0}^{t} \int_{0}^{t}\left(\Lambda\left(s_{1}\right) \Lambda\left(s_{2}\right)-1\right) \mathrm{d} \tilde{A}\left(b s_{1}\right) \mathrm{d} \tilde{A}\left(b s_{2}\right)\right) \\
& -\int_{0}^{t} \int_{0}^{t} \mathrm{E}\left(\Lambda\left(s_{1}\right) \Lambda\left(s_{2}\right)-1\right) \mathrm{d} s_{1} \mathrm{~d} s_{2} \\
& +\mathrm{E}\left(\frac{1}{b^{2}} \int_{0}^{t} \int_{0}^{t} \mathrm{~d} \tilde{A}\left(b s_{1}\right) \mathrm{d} \tilde{A}\left(b s_{2}\right)\right)-t^{2} \\
& +\int_{0}^{t} \int_{0}^{t} \mathrm{E}\left(\Lambda\left(s_{1}\right) \Lambda\left(s_{2}\right)-1\right) \mathrm{d} s_{1} \mathrm{~d} s_{2} \\
= & \frac{\sigma^{2}}{b^{2}} \int_{0}^{t} \int_{0}^{t} \rho\left(s_{1}, s_{2}\right) \operatorname{Cov}\left(\mathrm{d} \tilde{A}\left(b s_{1}\right), \mathrm{d} \tilde{A}\left(b s_{2}\right)\right)+\frac{1}{b^{2}} \operatorname{Var} A(b t) \\
& +\operatorname{Var}\left(\int_{0}^{t} \Lambda(s) \mathrm{d} s\right) .
\end{aligned}
$$

The claim is proved if we can show that both terms in the second last line are non-negative. Trivially, $\operatorname{Var} A(b t) \geq 0$. Since both $\Lambda$ and $A$ are positively correlated $\operatorname{Cov}\left(\mathrm{d} A\left(s_{1}\right), \mathrm{d} A\left(s_{2}\right)\right) \geq$ 0 and $\rho\left(s_{1}, s_{2}\right) \geq 0$. (It is straightforward to replace the somewhat heuristic infinitesimal covariance by a limit of finite increments).

Corollary 7. If $\int_{0}^{t} \Lambda(s) \mathrm{d}$ s is long range dependent then $A(t)$ is also.

\section{Examples}

In order to introduce an application friendly process family, i.e., parsimonious, causal, and easy to generate and analyze, we consider Markov jump processes which satisfy the following assumptions.

(i) $\Lambda^{(i)}(\cdot) \stackrel{\text { dist }}{=} \Lambda\left(b^{i} \cdot\right), \quad i=0,1, \ldots$, where $b>1$ and $\Lambda$ is a positive, stationary, positively correlated, piecewise constant Markov process.

(ii) The transition rates are bounded both above and below:

$$
\mathrm{P}(\Lambda(t) \text { constant on }[t, t+\Delta) \mid \Lambda(t)=x)=\exp (-\nu(x) \Delta),
$$

where $\nu_{\min } \leq \nu(x) \leq \nu_{\max }$ for all $x$ in the state space of $\Lambda$.

(iii) $A=\lim _{n \rightarrow \infty} A_{n}$ is non-degenerate. 
If we assume further that the variance of $\Lambda$ is finite and that its covariance decays exponentially fast ${ }^{2}$, then $b>1+\sigma^{2}$ is a sufficient condition for non-degeneracy of $A$ (by Corollary 2).

Proposition 7. In addition to (i)-(iii), assume $A \in \mathcal{L}_{q}$ and

$$
\mathrm{E} \sum_{T_{i} \in[0, t]} \Lambda\left(T_{i}\right)^{q} \leq C t
$$

where $T_{i}$ are the (random) jump points of $\Lambda$. Then the conclusion of Proposition 5 holds, i.e., $\mathrm{E} A(t)^{q} \sim t^{q-\log \mathrm{E} \Lambda^{q}}$.

Proof. By assumption (ii),

$$
\operatorname{EE}\left(\mathbf{1}\{\text { at least one jump on }[0, t]\} \Lambda^{q}(0) \mid \Lambda(0)\right) \leq\left(1-e^{-\nu_{\max } t}\right) \mathrm{E} \Lambda^{q} \leq C t .
$$

On the other hand,

$$
\mathrm{E} \sup _{s \in[0, t]}\left|\Lambda(0)^{q}-\Lambda(s)^{q}\right| \leq \mathrm{E}\left(\mathbf{1}\{\text { at least one jump on }[0, t]\} \Lambda^{q}(0)+\sum_{T_{i} \in[0, t]} \Lambda\left(T_{i}\right)^{q}\right) .
$$

Thus $c\left(q, b^{-n}\right) \leq C b^{-n}$ if (13) holds.

Condition (13) is satisfied, for example, when $\Lambda$ is bounded or $\Lambda\left(T_{i}\right)$ 's are independent of $T_{i}$. Then

$$
\mathrm{E} \sum_{T_{i} \in[0, t]} \Lambda\left(T_{i}\right)^{q} \leq \mathrm{EE}\left(\sum_{T_{i} \in[0, t]} \Lambda\left(T_{i}\right)^{q} \mid\left\{T_{i}\right\}\right) \leq C_{q} \mathrm{E}(\# \text { jumps in }[0, t]) \leq \tilde{C} t,
$$

where the last inequality follows from assumption 2 .

Example 1. Consider a stationary two-state Markov process $\Lambda(t)$ with transition rates $\nu_{1}$ and $\nu_{2}$ on the state space $S=\left\{S_{1}, S_{2}\right\}$. In order to have $\mathrm{E}(\Lambda(t))=1$, the transition rates must satisfy the equation

$$
\frac{\nu_{2} S_{1}}{\nu_{1}+\nu_{2}}+\frac{\nu_{1} S_{2}}{\nu_{1}+\nu_{2}}=1
$$

The covariance is given by

$$
\operatorname{Cov}(\Lambda(t), \Lambda(s))=\sigma^{2} e^{-\left(\nu_{1}+\nu_{2}\right)|s-t|},
$$

\footnotetext{
${ }^{2}$ This is always true if the state space is finite.
} 
where

$$
\sigma^{2}=\frac{\nu_{2} S_{1}^{2}+\nu_{1} S_{2}^{2}}{\nu_{1}+\nu_{2}}-1
$$

Constructing a family $\left\{\Lambda^{(i)}\right\}$ from the mother process $\Lambda$ by changing time

$$
\Lambda^{(i)}(\cdot) \stackrel{\text { dist }}{=} \Lambda\left(b^{i} \cdot\right), \quad i=0,1, \ldots
$$

means that the processes $\Lambda^{(i)}, i=0,1, \ldots$, are independent two-state Markov processes with transition rates $b^{i} \nu_{1}$ and $b^{i} \nu_{2}$. A realization of this construction is seen in figure 2.

Although the values of the mother process $\Lambda$ are dependent on the arrival process, we may still apply Proposition 7 since the state space of $\Lambda$ is bounded. We conclude that

$$
T(q)=q-1-\log _{b} \mathrm{E} \Lambda^{q}=q-1-\log _{b}\left(\frac{\nu_{2} S_{1}^{q}+\nu_{1} S_{2}^{q}}{\nu_{1}+\nu_{2}}\right) .
$$

Example 2. Let the mother process $\Lambda$ be a piecewise constant process with $\operatorname{Exp}(\nu)$ distributed i.i.d. lengths of constant periods. For each interval we draw independently a random value $M$ from a common distribution satisfying $\mathrm{E}(M)=1$. Thus, the process $\Lambda^{(n)}(t)$ is a piecewise constant process whose covariance is given by

$$
\operatorname{Cov}\left(\Lambda^{(n)}(0) \Lambda^{(n)}(x)\right)=\operatorname{Var}(M) e^{-\nu b^{n}|x|}=\sigma^{2} \rho_{n}(x) .
$$

A realization is shown in figure 3. Notice that this process is a generalization of Mandelbrot's martingale analyzed in [18]. Instead of having a deterministic division of the interval, we split according to a Poisson process.

Since the values of the mother process $\Lambda$ are independent on the arrival process we may apply Proposition 7 and conclude that

$$
T(q)=q-1-\log _{b} \mathrm{E} \Lambda^{q}=q-1-\log _{b} \mathrm{E}^{q} .
$$

Formally, this is the same formula as the one for the Martingale of Mandelbrot.

Even though these two examples seem to be quite similar, there are some differences which can also be observed visually. The first process has some sort of periodic structure due to the fact that after drawing the initial state of a multiplier the only randomness is in the lengths of the constant periods. The second process is clearly burstier. The obvious reason is the unboundedness of the multipliers. 

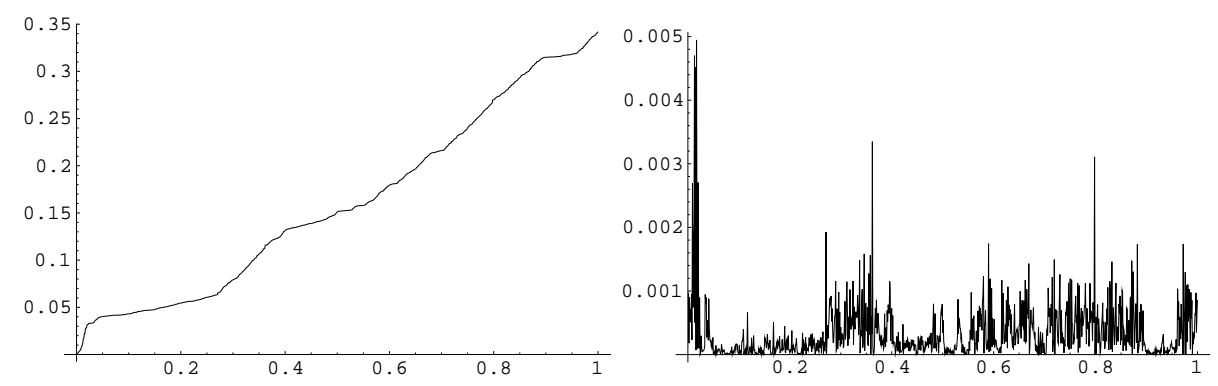

FIGURE 2: Example 1. On the left, a realization of process $A_{7}(t)$ with $\nu_{1}=2, \nu_{2}=1 / 2, S_{1}=1 / 3$, $S_{2}=7 / 6$ and $b=4$. On the right, the corresponding incremental process at resolution 0.001 .
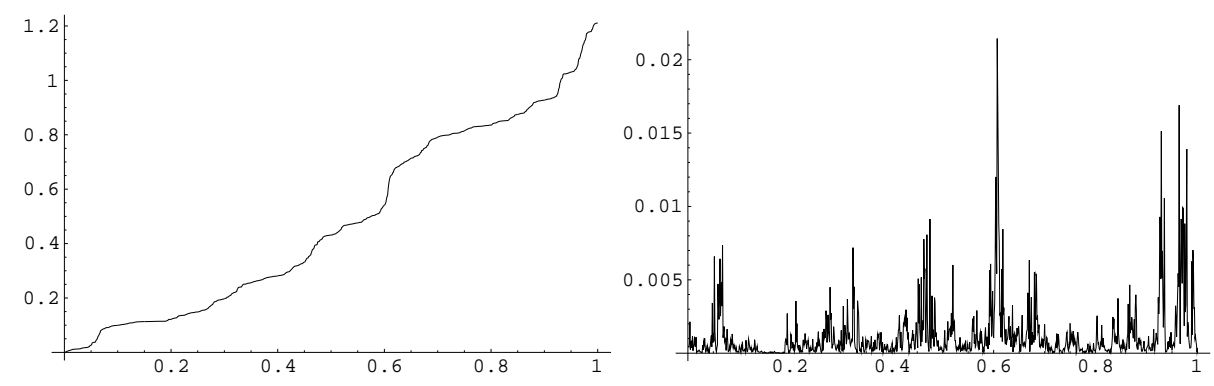

FIGURE 3: Example 2. On the left, a realization of process $A_{7}(t)$ with $\nu=1, b=4$, and $M \sim$ $\operatorname{Gamma}(3,1)$. On the right, the corresponding incremental process at resolution 0.001 .

\section{Concluding remarks}

The mathematical analysis of multifractal products of stochastic processes is far from complete. The aim of this paper was to give some basic definitions and properties in the general case, and show how this construction can be applied in the case of rescaled mother processes. The results given in this paper concern global behavior. A pathwise study of multifractal scaling properties involves a more delicate analysis of the almost sure local structure and is the object of current research.

\section{Acknowledgements}

The work of Petteri Mannersalo was supported by Academy of Finland, project 42535 . Rolf Riedi is grateful to the Academy of Finland for generous support during the summer school in Lahti, June 1999, during which the foundations of this work was laid. Financial support for 
Rolf Riedi comes from NSF grant No. ANI-00099148, DARPA/AFRL grant F30602-00-20557 and Texas Instruments.

\section{References}

[1] Arbeiter, M. (1991). Random recursive construction of self-similar fractal measures. the non-compact case. Probab. Th. Rel. Fields 88, 497-520.

[2] Arbeiter, M. and Patzchke, N. (1996). Random self-similar multifractals. Mathematiche Nachrichten 181, 5-42.

[3] Barral, J. (1999). Moments, continuité, et analyse multifractale des martingales de Mandelbrot. Probab. Theory Related Fields 113, 535-569.

[4] Burd, G. AND Waymire, E. (2000). Independent random cascades on Galton-Watson trees. Proceedings of the AMS 128, 2753-261.

[5] FALCONER, K. (1994). The multifractal spectrum of statistically self-similar measures. Journal of Theoretical Probability 7, 681-702.

[6] Fan, A. (1997). Sur le chaos de Lévy d'indice $0<\alpha<1$. Ann. Sc. Math. Québec 21, $53-66$.

[7] Fan, A. And Kahane, J. (2001). How many intervals cover a point in a random dyadic covering? Portugaliae Mathematica 58, 59-75.

[8] FAn, A. AND ShIEH, N. (2000). Multifractal spectrum for some random Gibbs measures. Stat. Prob. Let. 47, 25-31.

[9] Feldmann, A., Gilbert, A. And Willinger, W. (1998). Datanetworks as cascades: Investigating the multifractal nature of Internet WAN traffic. In Proceedings of the ACM/SIGCOMM'98. Vancouver, Canada.

[10] Gupta, V. AND Waymire, E. (1990). Multiscaling properties of spatial rainfall and river flow distributions. Journal of Geophysical Research 95(D3), 1999-2009.

[11] Holley, R. AND Waymire, E. (1992). Multifractal dimensions and scaling exponents for strongly bounded random cascades. Ann. Appl. Probab. 2, 819-845. 
[12] JafFard, S. (1996). Sur la nature multifractale des processus du Lévy. C. R. Acad. Sci. Paris 323, 1059-1064.

[13] Jaffard, S. (1999). The multifractal nature of Lévy processes. Probab. Theory Relat. Fields 114, 207-227.

[14] Kahane, J. (1989). Random multiplications, random coverings and multiplicative chaos. In Analysis at Urbana I. ed. E. Berkson, N. Peck, and J. Uhl. No. 137 in London Math. Soc. Lecture Notes. Cambridge University Press pp. 196-255.

[15] Kahane, J. (2000). Random coverings and multiplicative processes. In Fractal Geometry and Stochastics II. ed. C. Bandt, S. Graf, and M. Zähle. vol. 46 of Progress in Probability. Birkhäuser pp. 125-146.

[16] Kahane, J.-P. (1985). Sur le chaos multiplicatiff. Ann. Sc. Math. Québec 9, 105-150.

[17] Kahane, J.-P. (1987). Positive martingales and random measures. Chinese Annals of Mathematics 8B, 1-12.

[18] Kahane, J.-P. and Peyriére, J. (1976). Sur certaines martingales de Benoit Mandelbrot. Advances in Mathematics 22, 131-145.

[19] LÉVy VÉHel, J. AND RIEDI, R. (1997). Fractional Brownian motion and data traffic modeling: The other end of the spectrum. In Fractals in Engineering 97. Springer. pp. 185-202.

[20] Mandelbrot, B. (1972). Possible refinement of the lognormal hypothesis concerning the distribution of energy dissipation in intermittent turbulence. In Statistical models and turbulence. ed. M. Rosenblatt and C. Van Atta. No. 12 in Lecture notes in physics. Springer pp. 331-351.

[21] Mandelbrot, B. (1974). Intermittent turbulence in self-similar cascades: divergence of high moments and dimension of the carrier. Journal of Fluid Mechanics 64,.

[22] Mannersalo, P. AND Norros, I. (1997). Multifractal analysis of real ATM traffic: a first look. COST257TD(97)19. VTT Information Technology.

[23] Molchan, G. (1996). Scaling exponents and multifractal dimensions for independent random cascades. Communications in Mathematical Physics 179, 681-702. 
[24] Molchan, G. (2002). Mandelbrot cascade measures independent of branching parameter. Journal of Statistical Physics 107, 977-988.

[25] Patzchke, N. (1997). Self-conformal multifractal measures. Advances in Applied Mathematics 19, 486-513.

[26] Peyriére, J. (1977). Calculs de dimensions of Hausdorff. Duke Mathematical Journal 44, 591-601.

[27] Riedi, R., Crouse, M., Ribeiro, V. and Baraniuk, R. (1999). A multifractal wavelet model with application to network traffic. IEEE Transactions on Information Theory 45, 992-1018.

[28] Riedi, R. AND LÉVy VÉHEL, J. (1997). TCP traffic is multifractal: a numerical study. Inria research report, no. 3129. Project Fractales, INRIA Rocquencourt.

[29] Roberts, J., Mocci, U. And Virtamo, J., Eds. (1997). Broadband network teletraffic vol. 1155 of Lecture notes in computer science. Springer, Berlin.

[30] Waymire, E. And Williams, S. (1995). Multiplicative cascades: dimension spectra and dependence. J. Fourier Anal. \& Appl. 589-609. Special Issue in Honor of J.-P. Kahane.

[31] Waymire, E. And Williams, S. (1996). A cascade decomposition theory with applications to Markov and exchangeable cascades. Transactions of the AMS 348, 585631.

[32] Waymire, E. And Williams, S. (1997). Markov cascades. In Classical and Modern Branching Processes. ed. K. Athreya and P. Jagers. vol. 84 of The IMA Volumes in Mathematics and its Applications. Springer pp. 305-321. 